\title{
Title: Active Template Strategy for the Preparation of Interlocked Nanocarbons
}

Authors: James H. May ${ }^{1}$, Jeff M. Van Raden ${ }^{1}$, Ruth L. Maust ${ }^{1}$, Lev N. Zakharov ${ }^{2}$, Ramesh Jasti*

\section{Affiliations:}

${ }^{1}$ Department of Chemistry and Biochemistry, Materials Science Institute, and Knight

Campus for Accelerating Scientific Impact; University of Oregon, Eugene, Oregon 97403, United States

${ }^{2}$ CAMCOR - Center for Advanced Materials Characterization in Oregon; University of Oregon, Eugene, Oregon 97403, United States

*Corresponding author. Email: rjasti@uoregon.edu

Abstract: Mechanically interlocked carbon nanostructures represent a relatively unexplored frontier in carbon nanoscience due to the difficulty in preparing these unusual topological materials. Here we illustrate an active template method in which the key mechanical bond forming step is accomplished by leveraging unique ligand motifs to catalyze a cross-coupling reaction within the pore of a macrocycle. Once the mechanical bond has been formed, these macrocyclic ligands can then be converted into pi-conjugated structures in a subsequent synthetic step. This method provides a general strategy by which a variety of mechanically interlocked carbon nanostructures can be prepared which will enable structure-property relationships to be established for this emerging class of nanomaterials.

One-Sentence Summary: A bottom-up synthetic method is developed to synthesize new topological carbon nanomaterials.

\section{Main Text:}

The remarkable optical and electronic properties of carbon nanostructures are linked to their precise 3D arrangement of carbon atoms. Graphene, fullerene, and carbon nanotubes, while sharing very similar local bonding environments (i.e. exclusively $\mathrm{sp}^{2}$-hybridized bonded carbon), display vastly different physical properties due to the differing topologies of the overall structure (1). In recent years, using bottom-up organic synthesis strategies, new types of carbon nanostructures can be prepared that were inaccessible by more traditional thermodynamically driven methods. For example, substructures of CNTs, often referred to as carbon nanohoops (2) and carbon nanobelts (3-5), can now be prepared in which the size ( 6$)$, connectivity (7), and even heteroatom doping $(8)$ can be controlled with atomic precision. These molecular nanocarbons are gaining traction for a wide array of possible applications in organic electronics, biology and polymer science (9-13). A particularly exciting avenue is to use organic synthetic methods to prepare topologically unique carbon nanomaterials - in particular, mechanically interlocked structures. In 2019, Itami et al. reported the first syntheses of catenanes and a trefoil $40 \quad$ knot composed entirely of para-linked phenylene units using a traceless silyl tethering strategy (Fig. 1A) (14). The catenated and knotted molecules are structurally related to the 
$[n]$ cycloparaphenylenes ([n]CPPs), but the unique topologies of these structures give rise to dynamic motion and optical characteristics that differ from the parent $[n]$ CPPs. General synthetic methods to access a wide array of interlocked carbon nanostructures where the structures can be altered via connectivity, size, and heteroatom doping would bring about a new class of dynamic nanomaterials with engineerable optical and electronic properties. Herein, we disclose a versatile active template methodology that can deliver an array of mechanically interlocked nanocarbons of the catenane and rotaxane type, composed entirely of pi-conjugated units (Fig. 1B).

In 2006, Leigh and co-workers introduced the active template (AT) approach to mechanically interlocked molecules (MIMs) (15). In contrast to more common passive-template (PT) approaches $(16,17)$, the templating moiety in this case is actively involved in the formation of the mechanical bond. Specifically, the AT approach relies on a macrocycle to coordinate a catalytic metal ion endotopically (i.e. at the interior of its cavity). Bond forming reactions then are facilitated by this metal resulting in a product molecule that is threaded through the center of the macrocycle. Provided the threaded species is appropriately functionalized with large 'stopper' groups at either end, the 'thread' unit becomes mechanically entrapped and unable to diffuse away from the macrocycle. AT offers certain advantages over PT in that the breadth of structures that may be formed are not dictated by the inherent molecular recognition between interlocking components, but by the catalytic abilities of the templating metal-ion. Indeed, since the initial report by Leigh, Goldup and others have shown the remarkable structural diversity and range of applications enabled by this strategy (18).

Macrocycles with radial pi-systems, such as CPPs, however, are challenging motifs to use in AT approaches due to their lack of inwardly directed functional groups that might facilitate the required endotopic metal coordination (19-22). We envisioned a new approach in which macrocyclic CPP precursors are used for the AT reaction rather than the final aromatized structures. The methods developed in our lab for synthesizing CPPs center on the use of cyclohexadiene motifs as bent units that allow for the construction of low-strain macrocyclic precursors, which are ultimately converted to pi-conjugated molecules via reductive aromatization (2). Advantageously, these precursors have been prepared with wide ranging structural diversity, incorporating non-benzenoid aromatics, heterocycles, and even antiaromatic molecules $(8)$. We envisioned that these same cyclohexadiene systems might also provide the basis of a novel ligand motif that could enable CPP precursors to endotopically coordinate metalions. We hypothesized that macrocyclic structures that contain a cyclohexadiene motif with two flanking pyridine rings would provide a general active templating system to prepare a wide array of pi-conjugated, interlocked structures (Fig. 1C).

To assess the viability of this approach, pyridine containing macrocycles $4 \mathbf{a}$ and $\mathbf{4 b}$ (Fig. 2) were prepared in high yields and on the gram and half-gram scale respectively (see SI). Half-axel thread components were designed to be analogous to commonly used synthons in CPP syntheses but carrying a terminal alkyne (5a) or alkyne bromide (5b) functional group for the $\mathrm{Cu}^{\mathrm{I}}$ catalyzed Cadiot-Chodkiewicz cross-coupling. The AT Cadiot-Chodkiewicz (AT-CC) reaction was chosen for this study as it is well established in MIM syntheses and allows for the creation of fully piconjugated thread molecules (23-25). Moreover, if so desired, the resultant diyne is easily converted to other pi-conjugated units. We anticipated that the triethylsilyl (TES) ether substituents of $\mathbf{5 a}$ and $\mathbf{5 b}$ would provide sufficient steric bulk to serve as stopper units to prevent dethreading of the internally cross-coupled species. Accordingly, we envisioned accessing [2]rotaxane molecules that may serve as precursors to catenated structures consisting of a 
$\operatorname{diaza}[n] \mathrm{CPP}$ and an $[n+2] \mathrm{CPP}$, where the +2 of the latter denotes the presence of the alkyne units of the 1,4-butadyine moiety embedded in the CPP backbone.

Gratifyingly, both macrocycles $\mathbf{4 a}$ and $\mathbf{4 b}$ efficiently template the AT-CC reaction to afford [2] rotaxanes $6 \mathbf{a}$ and $\mathbf{6 b}$ in $65 \%$ and $53 \%$ yield respectively, provided excess ( $2.5 \mathrm{eq})$ of the coupling partners (5a, 5b) are used (Fig. 2). [2]Rotaxanes, $6 \mathbf{a}$ and $\mathbf{6 b}$, are subsequently transformed to the corresponding bis-boronates, $\mathbf{6 c}$, and $\mathbf{6 d}$, via a Miyaura borylation reaction, which, under dilute Suzuki-Miyaura cross coupling conditions reacts with S11, affording [2]catenanes $7 \mathbf{a}$ and $\mathbf{7 b}$ in $74 \%$ and $50 \%$ yield respectively. Subjecting $\mathbf{6 c}$ to similar macrocyclization reaction conditions using alternative coupling partner S12 afforded [2]catenane $7 \mathbf{c}$ in $58 \%$ yield. This demonstrates the modularity of these methods whereby [2] catenanes may be accessed in which the size of both the templating macrocycle and the secondary macrocycle formed in the last step may be varied. Aromatization reactions were performed via conditions adapted from those developed by Yamago et al. (26) to provide $\mathbf{1 a}, \mathbf{1 b}$, and $\mathbf{1 c}$ in $7 \%, 11 \%$, and $6 \%$ yield. While the yields for these final transformations are low, weighable quantities of the final compounds, sufficient for their characterization, could be obtained. In an attempt to access higher order interlocked molecules, 6a and $\mathbf{6}$ were subjected to dilute Suzuki-Miyaura crosscoupling to target a [3]catenane. This reaction, however, resulted only in a complex mixture of oligomeric products. Analysis of a space filling representation of the target molecule reveals (See SI) a large degree of steric congestion created by clashing TES groups of the two pre-existing macrocycles, likely precluding macrocyclization and favoring oligomer formation. To circumvent these issues, analogous [2]rotaxane synthons, $6 \mathbf{e}$ and $\mathbf{6 f}$, were prepared using the templating macrocycle $\mathbf{4 c}$ in which the TES substituents on the lower half (as drawn) of the macrocycle are replaced with smaller methyl ether groups. Satisfyingly, subjecting $\mathbf{6 e}$ and $\mathbf{6 f}$ to macrocyclization reaction conditions afforded [3] catenane, $7 \mathbf{d}$, in modest yield (39\%). Conversion to the aromatized structure was accomplished via conditions analogous to those used for 1a-c, affording 2 in $6 \%$ yield. The resulting structure consists of a [12+4]CPP ('+4' now that there are two butadiyne moieties present) threading two diaza[8]CPPs and, to our knowledge, represents the first [3] catenane to be made up of entirely pi-conjugated components.

Single crystals suitable for X-ray analysis were grown for 1a (Fig. 3A), 1b (Fig. 3B), and 2 (Fig. 3C), further confirming their catenated structures. In the solid-state, 1a exists as a dimer in which the diaza[8]CPP of one catenane occupies the void space of the $[11+2] \mathrm{CPP}$ from its dimeric partner. Increasing the size of the diaza $[n] \mathrm{CPP}$ by one phenylene unit prevents this dimer formation as evidenced by the solid-state structure of $\mathbf{1 b}$ which displays a much more open packing motif. In contrast to the structures of $\mathbf{1 a}$ and $\mathbf{1 b}$, the diaza[8]CPPs of $\mathbf{2}$ reside over the two opposing butadiyne moieties of the mutually encapsulated $[12+4] \mathrm{CPP}$. This shift in preference is likely do to the sterically congested nature of the structure in which the most efficient separation of mass is achieved with the two diaza[8]CPPs existing along the major axis of the $[12+4] \mathrm{CPP}$ 's elliptical structure. Indeed, the structure of the diyne containing ring contorts significantly in accommodation of the two interlocking rings, displaying impressively acute -(C$\mathrm{C} \equiv \mathrm{C})$ - angles as low as $163.8^{\circ}\left(164.8^{\circ}\right.$ and $164.1^{\circ}$ for $\mathbf{1 a}$ and $\mathbf{1 b}$ respectively). The solid-state packing of organic molecules is directly correlated to useful materials properties such as chargetransport characteristics (27), as well as their utility as porous molecular materials (28). The introduction of mechanical linkages represents a unique approach to influence the crystal packing of CPPs beyond merely adjusting the diameter or atomic composition. Interestingly, many close contacts $(\leq 3.5 \AA)$ were observed in the crystal structures of 1a-c and $\mathbf{2}$ when compared to the crystal structures of diaza[8]CPP and diaza[9]CPP. Not only do the catenated structures show a greater number of these contacts, but the mean distance between contacting 
atoms is markedly lower. This is most dramatic in the case of 1a (Fig 3D, left) in which 14 distinct intermolecular short contacts $(\leq 3.5 \AA)$ are observed between non-bonded carbon (or nitrogen) atoms of a catenane and its neighbors. This is in stark contrast to the structure of diaza[8]CPP in which only two contacts exist that are $\leq 3.5 \AA$ (Fig 3D, right), illustrating the large influence that catenation may have on the materials properties of molecular nanocarbons.

Encouraged by our success in synthesizing catenated molecules, we envisioned that [2] rotaxane precursors similar to 6a-f might also be used as intermediates in the synthesis of higher order $[n]$ rotaxanes $(n>2)$ featuring fully conjugated threads. To investigate, [2]rotaxanes, $\mathbf{6 g}$ and $\mathbf{6 h}$, were prepared (Fig. 4) in which ${ }^{t} \mathrm{Bu}$-substitued trityl groups are installed on one side of the thread. This bulky substituent serves as a permanent stopper that remains at the thread termini following the removal of silyl groups. [3]Rotaxane, 8, is readily prepared by the Suzuki crosscoupling of $\mathbf{6 g}$ and $\mathbf{6 h}$ in $92 \%$ yield which upon reductive aromatization provides [3] rotaxane $\mathbf{3}$ in $5 \%$ yield. Remarkably, despite the 14 unsubstituted phenyl rings and two interspacing butadiene moieties that make up the thread, $\mathbf{3}$ was completely soluble in common organic solvents and could be characterized by ${ }^{1} \mathrm{H}$ and ${ }^{13} \mathrm{C}$ NMR. Given the high solubility of $\mathbf{3}$, one might imagine that [2] rotaxanes analogous to $6 \mathbf{a}$ and $\mathbf{6 g}$ could serve as monomer and endcapping units, respectively, in the formation of insulated pi-conjugated wires, offering a new route to this class of electronic material. As it stands, the yields for the final reductive aromatization reaction are prohibitively low for such a pursuit, however, future work will seek alternative reaction conditions that provide more efficient conversion to the aromatized structures.

To examine the impact of the mechanical bond on the photophysical properties of these molecules, we independently synthesized and characterized control molecules diaza[8]CPP, diaza[9]CPP along with [11+2]CPP and [12+2]CPP. As shown in Fig. 5, the photophysical properties of these molecules are consistent with parent CPPs, where the UV-Vis max absorption is independent of size $\left(\lambda_{\max } \sim 345 \mathrm{~nm}\right)$, but the emission maximum redshifts with decreasing size (e.g. diaza[8]CPP is redshifted versus diaza[9]CPP) (29). The photophysical characteristics of 1a-c, 2 and $\mathbf{3}$ were also assessed via UV-Vis and fluorescence spectroscopy (Fig. 5). The UV-Vis absorbance profiles of all the interlocked structures are nearly identical to the control molecules, each having a $\lambda_{\max } \sim 345 \mathrm{~nm}$. In contrast, the emission profiles of the interlocked structures vary depending on the exact composition. In each case, the dominant emission is that of the smaller $\operatorname{diaza}[n] \mathrm{CPP}$, with 1c having an emission maximum centered around $509 \mathrm{~nm}$ and $\mathbf{1 a - b}, \mathbf{2}$, and $\mathbf{3}$ having an emission maximum centered around $550 \mathrm{~nm}$. In addition to the emission from the $\operatorname{diaza}[n] \mathrm{CPP}$, the fluorescence profiles of each interlocked structure also display a weak blue shoulder stemming from the emission of the diyne containing CPPs (or linear thread component in the case of $\mathbf{3}$ ). As the brightness of CPP emission is known to increase with increasing ring size (29), the rather modest contribution of the diyne containing CPP to the catenanes' overall fluorescence profile is consistent with energy transfer from the larger hoop to the smaller hoop (14). The further study of the photophysics of these dynamic catenane and rotaxane systems is an exciting prospect for the future.

Very few types of macrocycles have been used in AT chemistry (18), typically being flexible ethereal macrocycles with embedded phenanthrene or bipyridine motifs. Owing to the unusual nature of macrocyclic ligands $\mathbf{4 a}$ and $\mathbf{4 b}$, we sought to gain a better understanding of the interaction of these molecules with the $\mathrm{Cu}$ catalyst. Specifically, we were curious to whether both pyridine rings are required to direct reactivity toward the interior of the macrocycle as we had originally designed. To test this, macrocycle S15 was synthesized, which is identical to 4a, 
but features only a single pyridine ring. Interestingly, no interlocked products were observed for AT-CC reactions with $\mathbf{S 1 5}$, which is consistent with both pyridines acting cooperatively in the endotopic binding of the $\mathrm{Cu}$ ion. We were also interested whether alternative ligand geometries could be amenable to AT chemistry. To probe this, macrocycle $\mathbf{4 d}$ was synthesized which, like 4a, carries two nitrogen atoms but placed at different positions along the macrocycle.

Surprisingly, 4d outperforms $\mathbf{4 a}$ in the AT-CC reaction, providing [2] rotaxane $\mathbf{6 i}$ in $82 \%$ yield. While it seems that both pyridine rings are essential in sequestering the $\mathrm{Cu}$ ion within the pore of the macrocycle, the success of the AT-CC reaction using $\mathbf{4 d}$ suggests that a bidentate binding model is likely an oversimplification of a more complex/dynamic interaction(s). This is significant as it demonstrates that macrocycles with atypical ligand motifs may be effectively employed in AT chemistry. As such, we anticipate that a wider array of macrocyclic motifs might be made suitable for AT chemistry than were previously appreciated. Further mechanistic investigations are warranted to fully understand the nature of these unusual ligands.

In summary, we report a new active template synthesis by which mechanically interlocked CPPs of varying substructure and topology may be prepared. The methods disclosed in this report dramatically increase the structural space that may be explored in the context of topological nanocarbon species and lays the synthetic groundwork for the preparation of structures of even greater complexity than those presented here. Importantly, the synthetic method leverages the well-established building block approach to nanohoop architectures, which is already being employed by numerous research groups. While we have confirmed the validity of this approach for the precursors of diaza[8]CPP and diaza[9]CPP, we anticipate that both smaller and larger macrocycles, bearing these ligand motifs, would be capable of participating in the AT-CC reaction allowing for a detailed analysis of the influence of relative ring size on the properties of nanohoop catenanes. Further, owing to the simplicity of the mechanical bond forming reaction, we envision that this AT methodology may be adapted to produce a wide array of interlocked structures including those decorated with functional groups or even extended polymeric materials. We anticipate that the generality of this approach will provide access to topological carbon nanostructures with emergent optical and electronic characteristics that were previously inaccessible.

\section{References and Notes}

1. S. Thomas, C. Sarathchandran, S. A. Ilangovan, J. C. Moreno-Pirajan, Handbook of carbon-based nanomaterials (Elsevier, ed. 1, 2021).

2. R. Jasti, J. Bhattacharjee, J. B. Neaton, C. R. Bertozzi, Synthesis, characterization, and theory of [9]-, [12]-, and [18]cycloparaphenylene: carbon nanohoop structures. J. Am. Chem. Soc. 130, 17646-17647 (2008).

3. G. Povie, Y. Segawa, T. Nishihara, Y. Miyauchi, K. Itami, Synthesis of a carbon nanobelt. Science (80-. ). 356, 172-175 (2017).

4. K. Y. Cheung, S. Gui, C. Deng, H. Liang, Z. Xia, Z. Liu, L. Chi, Q. Miao, Synthesis of Armchair and Chiral Carbon Nanobelts. Chem. 5, 838-847 (2019).

5. K. Y. Cheung, K. Watanabe, Y. Segawa, K. Itami, Synthesis of a zigzag carbon nanobelt. Nat. Chem. 13, 255-259 (2021).

6. E. R. Darzi, T. J. Sisto, R. Jasti, Selective syntheses of [7]-[12]cycloparaphenylenes using orthogonal Suzuki-Miyaura cross-coupling reactions. J. Org. Chem. 77, 6624-6628 
(2012).

7. T. C. Lovell, C. E. Colwell, L. N. Zakharov, R. Jasti, Symmetry breaking and the turn-on fluorescence of small, highly strained carbon nanohoops. Chem. Sci. 10, 3786-3790 (2019).

8. M. Hermann, D. Wassy, B. Esser, Conjugated nanohoops incorporating donor, acceptor, hetero- or polycyclic aromatics. Angew. Chem. Int. Ed. 60, 15743-15766 (2021).

9. E. J. Leonhardt, R. Jasti, Emerging applications of carbon nanohoops. Nat. Rev. Chem. 3, 672-686 (2019).

10. B. M. White, Y. Zhao, T. E. Kawashima, B. P. Branchaud, M. D. Pluth, R. Jasti, Expanding the chemical space of biocompatible fluorophores: nanohoops in cells. ACS Cent. Sci. 4, 1173-1178 (2018).

11. T. C. Lovell, S. G. Bolton, J. P. Kenison, J. Shangguan, C. E. Otteson, F. Civitci, X. Nan, M. D. Pluth, R. Jasti, Subcellular targeted nanohoop for one- and two-photon live cell imaging. ACS Nano 15, 15285-15293 (2021).

12. G. M. Peters, G. Grover, R. L. Maust, C. E. Colwell, H. Bates, W. A. Edgell, R. Jasti, M. Kertesz, J. D. Tovar, Linear and radial conjugation in extended $\pi$-electron systems. $J$. Am. Chem. Soc. 142, 2293-2300 (2020).

13. Q. Huang, G. Zhuang, M. Zhang, J. Wang, S. Wang, Y. Wu, S. Yang, P. Du, A long $\pi$ conjugated poly(para-phenylene)-based polymeric segment of single-walled carbon nanotubes. J. Am. Chem. Soc. 141, 18938-18943 (2019).

14. Y. Segawa, M. Kuwayama, Y. Hijikata, M. Fushimi, T. Nishihara, J. Pirillo, J. Shirasaki, N. Kubota, K. Itami, Topological molecular nanocarbons: All-benzene catenane and trefoil knot. Science 365, 272-276 (2019).

15. V. Aucagne, K. D. Hänni, D. A. Leigh, P. J. Lusby, D. B. Walker, Catalytic "click" rotaxanes: A substoichiometric metal-template pathway to mechanically interlocked architectures. J. Am. Chem. Soc. 128, 2186-2187 (2006).

16. C. O. Dietrich-Buchecker, J. P. Sauvage, J. P. Kintzinger, Une nouvelle famille de molecules : les metallo-catenanes. Tetrahedron Lett. 24, 5095-5098 (1983).

17. G. Barin, A. Coskun, M. M. G. Fouda, J. F. Stoddart, Mechanically interlocked molecules assembled by $\pi-\pi$ recognition. Chempluschem. 77, 159-185 (2012).

18. M. Denis, S. M. Goldup, The active template approach to interlocked molecules. Nat. Rev. Chem. 1, 1-17 (2017).

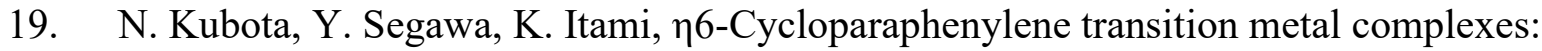
Synthesis, structure, photophysical properties, and application to the selective monofunctionalization of cycloparaphenylenes. J. Am. Chem. Soc. 137, 1356-1361 (2015).

20. J. M. Van Raden, S. Louie, L. N. Zakharov, R. Jasti, 2,2'-Bipyridyl-embedded cycloparaphenylenes as a general strategy to investigate nanohoop-based coordination complexes. J. Am. Chem. Soc. 139, 2936-2939 (2017).

21. J. M. Van Raden, B. M. White, L. N. Zakharov, R. Jasti, Nanohoop Rotaxanes from Active Metal Template Syntheses and Their Potential in Sensing Applications. Angew. 
Chemie - Int. Ed. 58, 7341-7345 (2019).

22. Y. Y. Fan, D. Chen, Z. A. Huang, J. Zhu, C. H. Tung, L. Z. Wu, H. Cong, An isolable catenane consisting of two Möbius conjugated nanohoops. Nat. Commun. 9, 3037 (2018).

23. J. Berná, S. M. Goldup, A. L. Lee, D. A. Leigh, M. D. Symes, G. Teobaldi, F. Zerbetto, Cadiot-Chodkiewicz active template synthesis of rotaxanes and switchable molecular shuttles with weak intercomponent interactions. Angew. Chem. Int. Ed. 47, 4392-4396 (2008).

24. M. J. Langton, J. D. Matichak, A. L. Thompson, H. L. Anderson, Template-directed synthesis of $\pi$-conjugated porphyrin [2] rotaxanes and a [4]catenane based on a sixporphyrin nanoring. Chem. Sci. 2, 1897-1901 (2011).

25. L. D. Movsisyan, M. Franz, F. Hampel, A. L. Thompson, R. R. Tykwinski, H. L. Anderson, Polyyne rotaxanes: stabilization by encapsulation. J. Am. Chem. Soc. 138, 1366-1376 (2016).

26. S. Hashimoto, E. Kayahara, Y. Mizuhata, N. Tokitoh, K. Takeuchi, F. Ozawa, S. Yamago, Synthesis and physical properties of polyfluorinated cycloparaphenylenes. Org. Lett. 20, 5973-5976 (2018).

27. V. Coropceanu, J. Cornil, D. A. da Silva Filho, Y. Olivier, R. Silbey, J. L. Brédas, Charge transport in organic semiconductors. Chem. Rev. 107, 926-952 (2007).

28. D. C. Milan, M. Krempe, A. K. Ismael, L. D. Movsisyan, M. Franz, I. Grace, R. J.

Brooke, W. Schwarzacher, S. J. Higgins, H. L. Anderson, C. J. Lambert, R. R. Tykwinski, R. J. Nichols, The single-molecule electrical conductance of a rotaxane-hexayne supramolecular assembly. Nanoscale 9, 355-361 (2017).

29. E. R. Darzi, R. Jasti, The dynamic, size-dependent properties of [5][12]cycloparaphenylenes. Chem. Soc. Rev. 44, 6401-6410 (2015). 


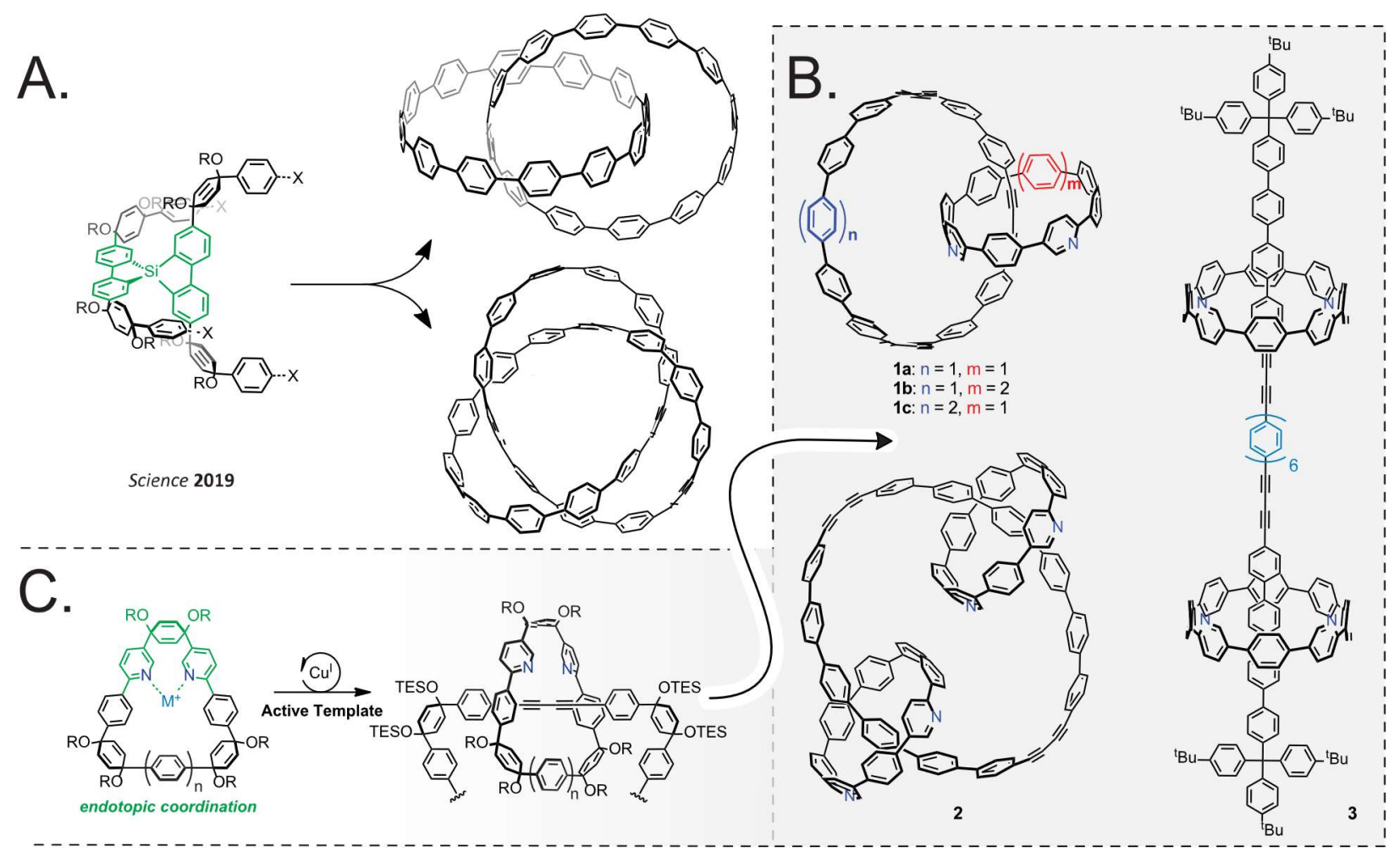

Fig. 1: (A) Covalent silicon tethering approach to all benzene catenane and trefoil knot. (B) Structures of catenanes 1a-c, $\mathbf{2}$ and [3] rotaxane 3. (C) general strategy to access title compound $\mathbf{1 a - c ,} \mathbf{2}$, and $\mathbf{3}$. 


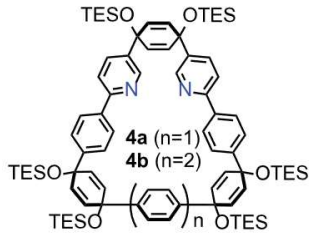

$\mathbf{5 a}, \mathbf{5 b}$
$\left[\mathrm{Cu}(\mathrm{MeCN})_{4}\right] \mathrm{PF} 6$
$(i \mathrm{Pr})_{2} \mathrm{NEt}$
$\mathrm{CHCl}_{3}, 50^{\circ} \mathrm{C}$

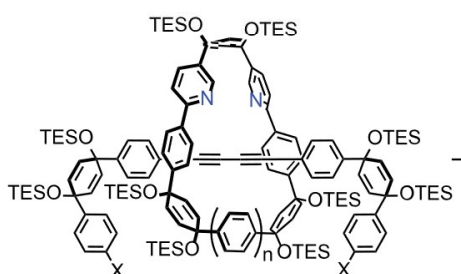

$\mathrm{B}_{2} \mathrm{pin}_{2}$
$\mathrm{Pd}(\mathrm{OAc})_{2}, \begin{aligned} & \mathbf{S P h o s} \\ & \mathrm{K}_{3} \mathrm{PO}_{4}\end{aligned}$
Dioxane, $80{ }^{\circ} \mathrm{C}(\mathrm{x}=\mathrm{Cl}, \mathrm{n}=1) 65 \%$
$\mathbf{6 b}(\mathrm{x}=\mathrm{Cl}, \mathrm{n}=2) 53 \%$
$\mathbf{6 c}(\mathrm{c}=\mathrm{Bpin}, \mathrm{n}=1) 98 \%$
$\mathbf{6 d}(\mathrm{x}=\mathrm{Bpin}, \mathrm{n}=2) 84 \%$
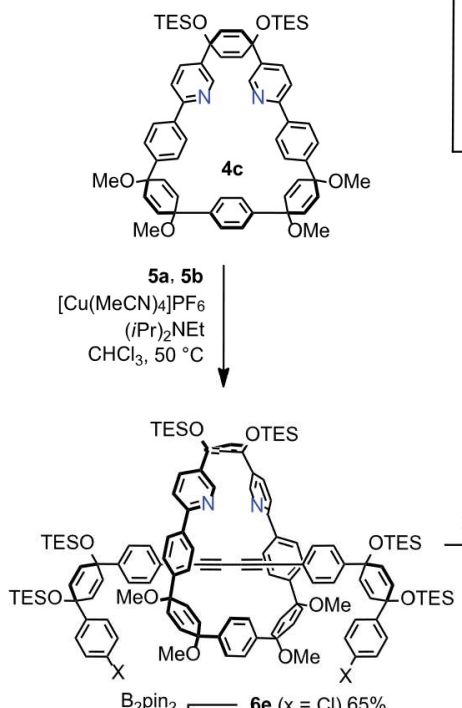

$\mathrm{Pd}(\mathrm{OAc})_{2}, \mathrm{SPhos}_{\mathrm{K}_{3} \mathrm{PO}_{4}}$
$\mathrm{~K}_{3} \mathrm{PO}_{4}$
Dioxane, $80{ }^{\circ} \mathrm{Ce}(\mathrm{x}=\mathrm{Cl}) 65 \%$
$\longrightarrow 6 \mathbf{6 f}(\mathrm{x}=\mathrm{Bpin}) 96 \%$
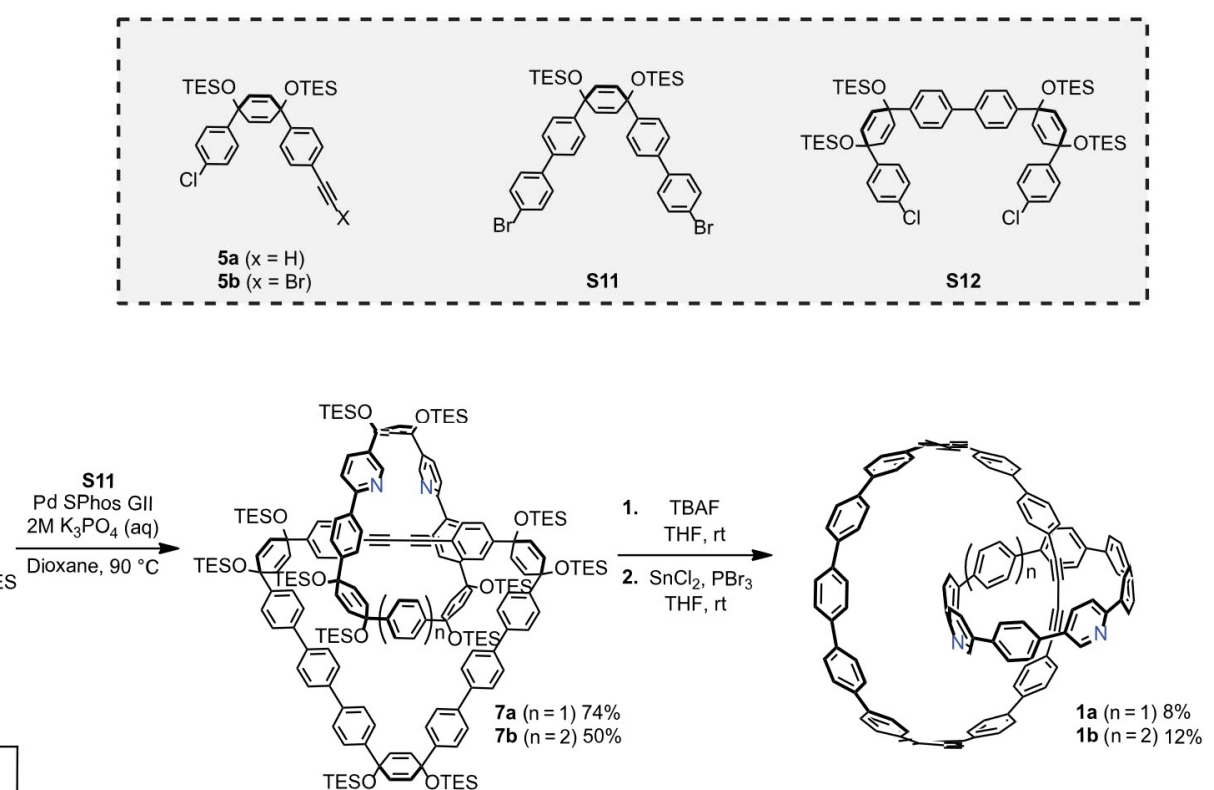

Fig. 2: Synthetic routes to catenanes $\mathbf{1 a - c}$, and $\mathbf{2}$. 

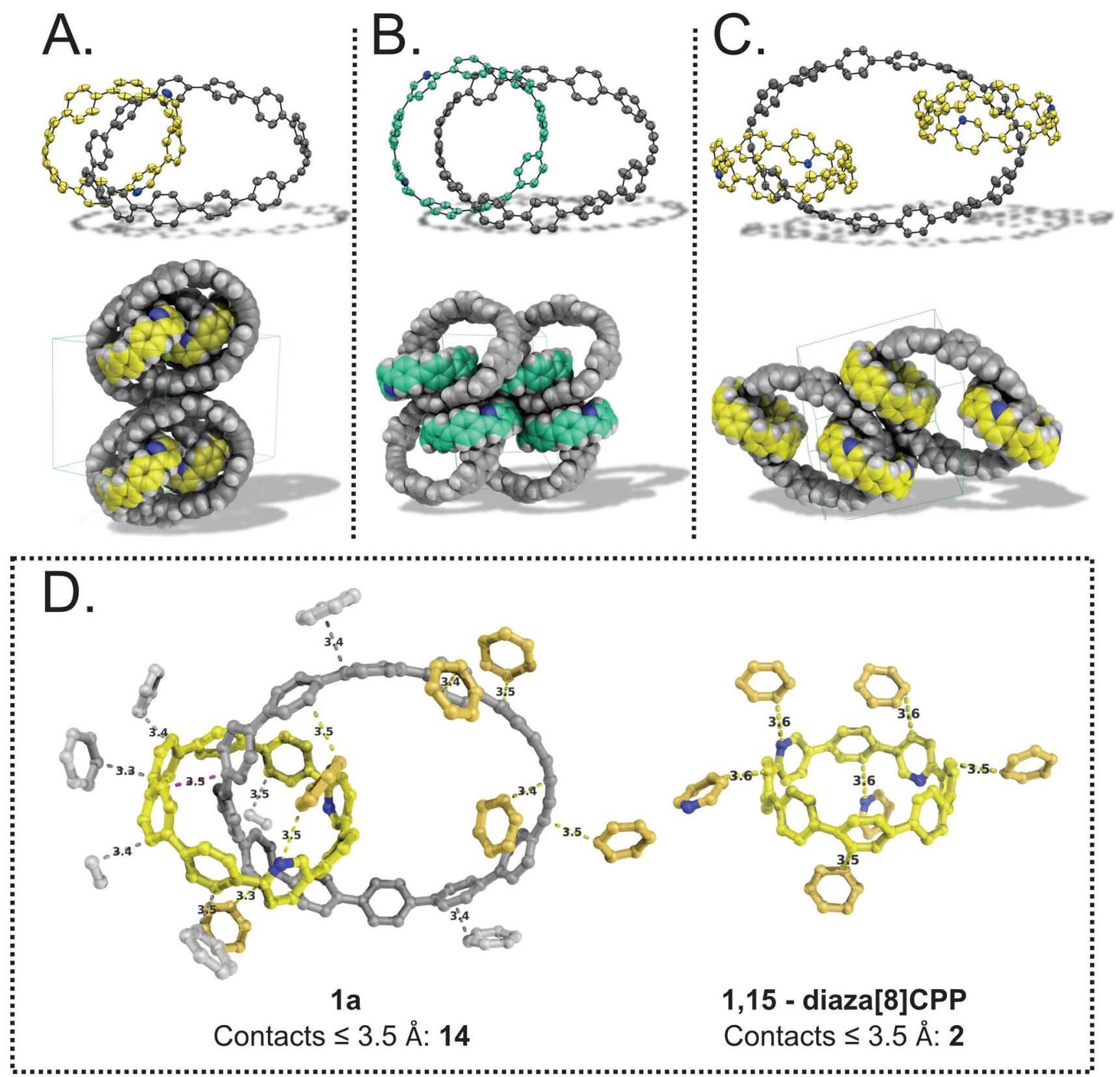

Fig. 3: X-ray structures of $\mathbf{1 a}, \mathbf{1 b}$, and $\mathbf{2}$. (A-C, top) Oakridge thermal ellipsoid (ORTEP) drawings of 1a (A), $\mathbf{1 b}$ (B), and $\mathbf{2}$ (C) with thermal ellipsoids set to $50 \%$ probability. Hydrogen atoms and solvent molecules have been omitted for clarity. (A-C, bottom) Packing structures of $\mathbf{1 a}(\mathbf{A}), \mathbf{1} \mathbf{b}(\mathbf{B})$, and $\mathbf{2}$ (C); carbon: grey, yellow, or green; hydrogen: white. Solvent molecules have been omitted for clarity. (D) short contacts observed in the X-ray structures of 1a (left) and diaza[8]CPP (right). Contacted fragments are colored to distinguish between those belonging to neighboring diaza[8]CPPs (orange) and neighboring [11+2]CPPs (grey). 

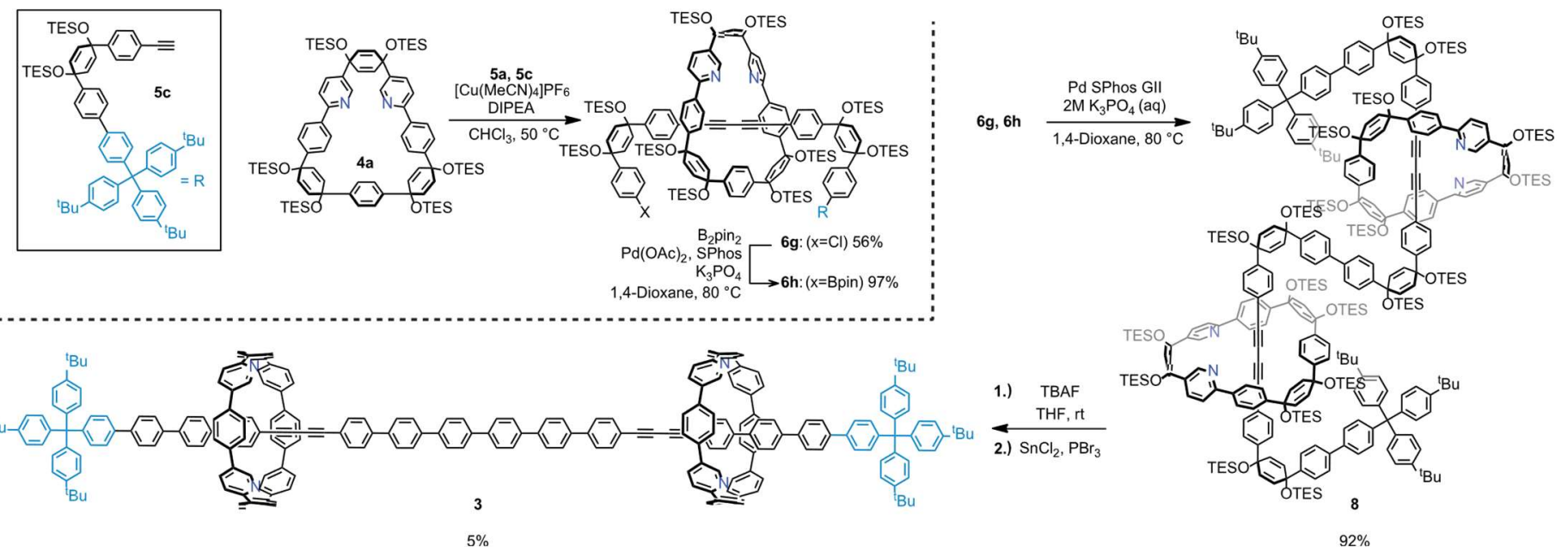

Fig. 4: Synthetic route to [3]rotaxane 3.

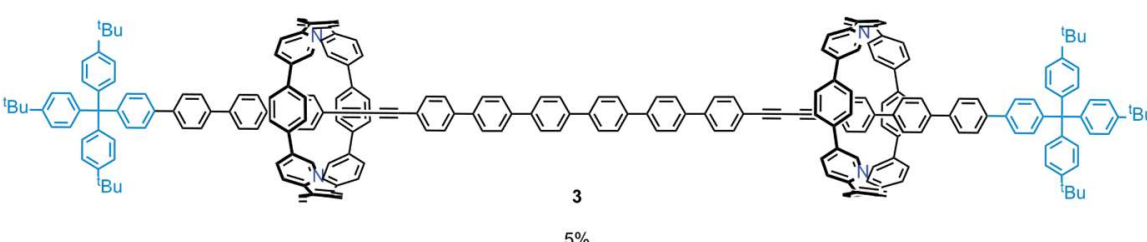

A.

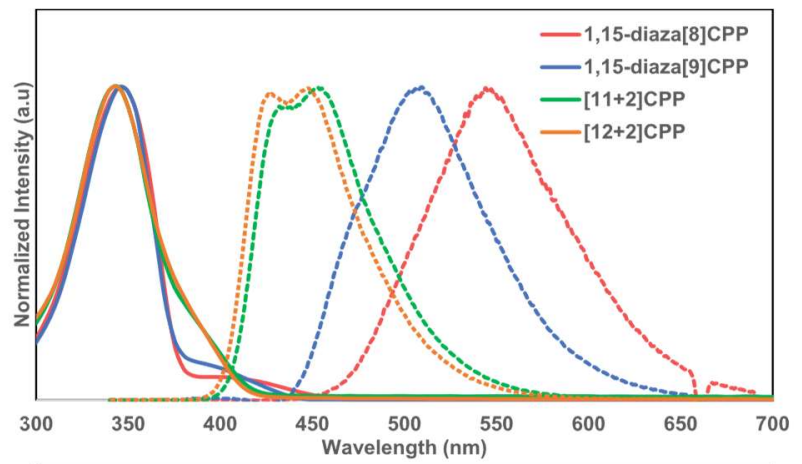

B.

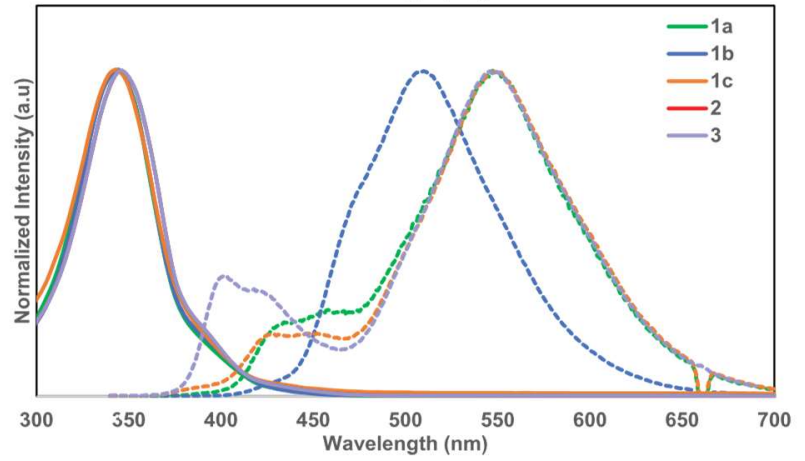

Fig. 5: (A) Ultraviolet-visible absorbance (solid lines) and fluorescence (dashed lines) spectra of interlocked structures 1a-c, 2, and 3. (B) Ultraviolet-visible absorbance (solid lines) and fluorescence (dashed lines) spectra of diaza[n]CPPs $(n=8,9)$ and $[n+2]$ CPPs $(n=11,12)$, free of mechanical linkage. 


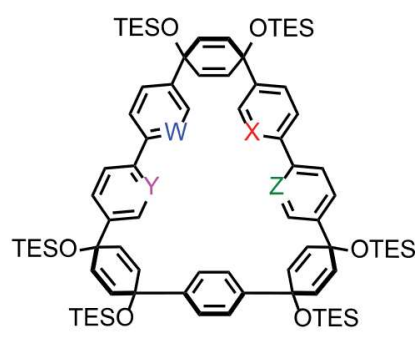

$4 a(W, X=N ; Y, Z=C H)$

4d $(\mathrm{W}, \mathrm{X}=\mathrm{CH} ; \mathrm{Y}, \mathrm{Z}=\mathrm{N})$

S15 $(W=N ; X, Y, Z=C H)$
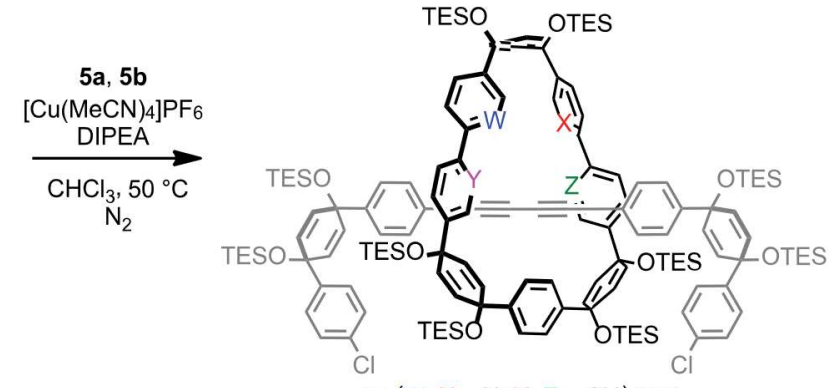

6a $(W, X=N: Y, Z=C H) 65 \%$

6i $(\mathrm{W}, \mathrm{X}=\mathrm{CH} ; \mathrm{Y}, \mathrm{Z}=\mathrm{N}) 82 \%$

SS1 $(W=N ; X, Y, Z=C H) 0 \%$

Fig. 6: Comparison of AT-CC reactions run with macrocyclic ligands 4a, 4d, and S8 along with the associated yield of the interlocked product. 